\title{
Innovation of Intelligent Tensioning in Precast Box Girder
}

\author{
Jianhao Zheng ${ }^{1}$, Hailong Zhang ${ }^{1}$, Jiawei Wang ${ }^{2 *}$ \\ ${ }^{1}$ Guangdong Zihui Expressway Co., Ltd, Huizhou, Guangdong, 516000, China \\ 2 Beijing Xinqiao Technology Development Co., Ltd, Beijing, 100088, China
}

\begin{abstract}
Because of the high precision and stability of the intelligent system, the intelligent tensioning technology can completely eliminate the interference of human factors and effectively ensure the construction quality of the prestressed tensioning. In this paper, the characteristics, principle, process flow and points for attention in the process of intelligent tensioning of precast box girder are introduced.
\end{abstract}

\section{Introduction}

The application of Prestressed Engineering in the bridge structure effectively improves the service performance of the upper structure of the bridge. If the prestressed tension construction is not standardized or lacks effective quality control means, the prestressed bridge will have greater quality and safety risks. Therefore, ensuring the quality of prestressed construction is the key link affecting the safety and durability of the bridge structure. In traditional construction, manual operation is adopted. Due to the uncontrollable limitation of monitoring means and human factors, there are some problems in prestressed tension, such as the synchronization accuracy can not be guaranteed, the tension control is not strict, the elongation measurement is not accurate, and the load holding time is not sufficient, which affect the quality of prestressed construction and seriously endanger the safety of bridge structure.

Due to the high precision and stability of the intelligent system, intelligent tensioning technology can completely eliminate the interference of human factors and effectively ensure the construction quality of prestressed tensioning.

- Intelligent control to standardize the tensioning process and ensure the safety of tensioning data;

- $\quad$ Easy to operate;

- Stress is applied accurately and elongation is checked in time to realize "double control";

- Automatic fault detection;

- Supervision audit and Internet view data function, convenient management.

In order to ensure that the quality of bridge prestressed construction meets the design and current specification requirements, the intelligent tensioning system is adopted for the prestressed construction of the prefabricated small box girder in the elevated part of Zihui expressway. By changing the original construction technology, the intelligent control of the whole tensioning process is realized, and the "real-time tracking, intelligent control and timely error correction" of the tensioning construction management is realized, which basically eliminates the manual tensioning The problems of low measurement accuracy and untrue data record in the process of pull-out can reduce the influence of human factors. While ensuring the construction quality of prestressed tensioning, it greatly improves the construction management level and efficiency, and ensures the safety and durability of bridge structure.

\section{Working Principle}

The intelligent tensioning equipment is composed of main engine, oil pump and jack.

Intelligent tension control system takes tension force as control index and elongation error as proofreading index. The system collects the working pressure and strand elongation (retraction of the force bar) of each jack by sensor induction measurement, and the data is transmitted to the host computer of the system in real time. After operation and judgment, the data is transferred to the PLC. The PLC adjusts the working parameters of the pump motor at any time, controls the oil output to get the required pressure value, so as to achieve high-precision tension and loading speed control. The system can also control each mechanical action of each device synchronously and complete the whole stretching process automatically by issuing instructions from the host according to the preset program.

\section{3 technical preparation}

\section{1 elastic modulus of steel strand}

The elastic modulus of steel strand is the influencing factor of tension elongation. During tension operation, the theoretical elongation value is adjusted according to the elastic modulus value of each batch of steel strand re inspection report, and the diameter of steel strand is checked to ensure the accuracy of tension parameters. 


\section{2 concrete strength and elastic modulus}

The elastic modulus and strength index of concrete have great influence on the crack control, tension safety and creep of beam body in the later stage. In order to reduce the prestress loss during and after the bridge prestress tension, the elastic modulus and strength of the concrete test block under the same condition corresponding to the prefabricated small box girder are tested before each tension to ensure that the measured value meets the design requirements.

\section{3 calculation of beam compression value during tension}

When calculating the compression of the beam body, it is considered when each beam is tensioned separately, and the cross-sectional area of the middle span of the beam body is taken as the calculation section.

\section{4 correction of theoretical elongation}

When calculating the theoretical elongation value, the influence of concrete strength, elastic modulus, beam compression and other factors are considered, and the correction is carried out to ensure the accuracy of the data.

\section{5 setting intelligent tensioning system}

Under the guidance of the intelligent tension system technicians, the software installation, system debugging and parameter setting are completed.

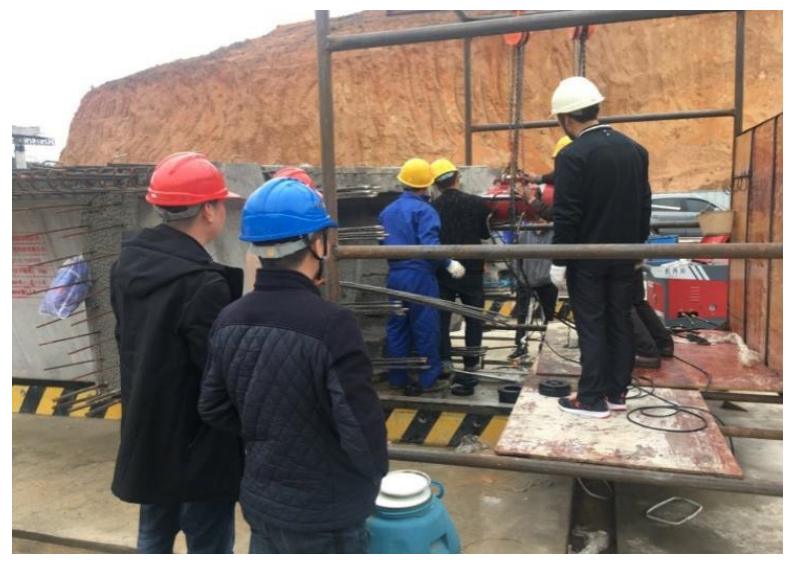

Figure 1. Intelligent tensioning field of precast box girder

\section{Construction process}

\subsection{Bellows installation}

Pre-stress is usually made of plastic bellows, which are installed strictly in accordance with the position and requirements of the design drawings and are securely fixed by positioning bars. One U-shaped erection bar is fixed in the straight section about $0.5 \mathrm{~m}$, and the curve section is encrypted to avoid displacement of the bellows during concrete placement.

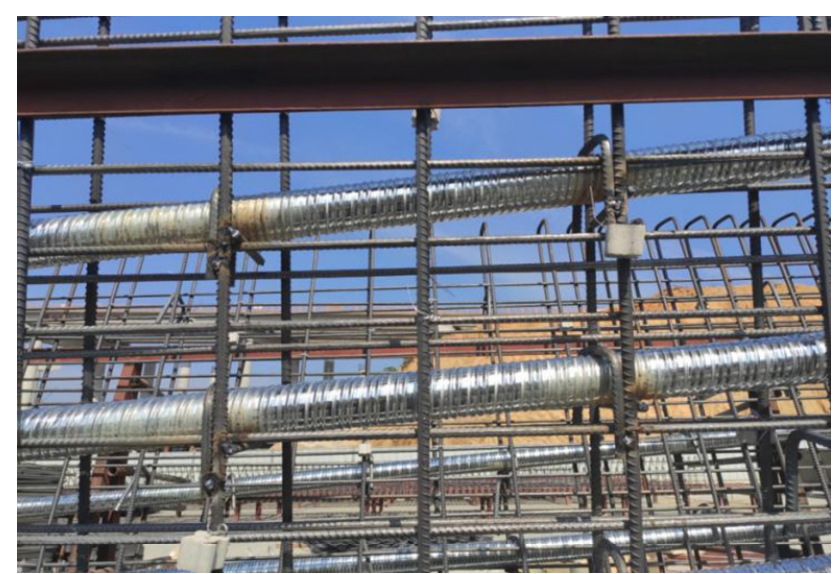

Figure 2. Bellows installation

\subsection{Anchor Plate Installation}

Install the corresponding type and specification anchor plate and screw bar at the tension end, and firmly connect the bottom of the horn opening of the anchor plate to the bellows. The anchor plate should be firmly installed on the template. When installing the anchor backing plate, for the anchorage with tension at both ends, it is necessary to pay attention to the fact that the grouting inlet hole at the grouting end is down and the outlet hole is up so as to ensure the density of grouting.

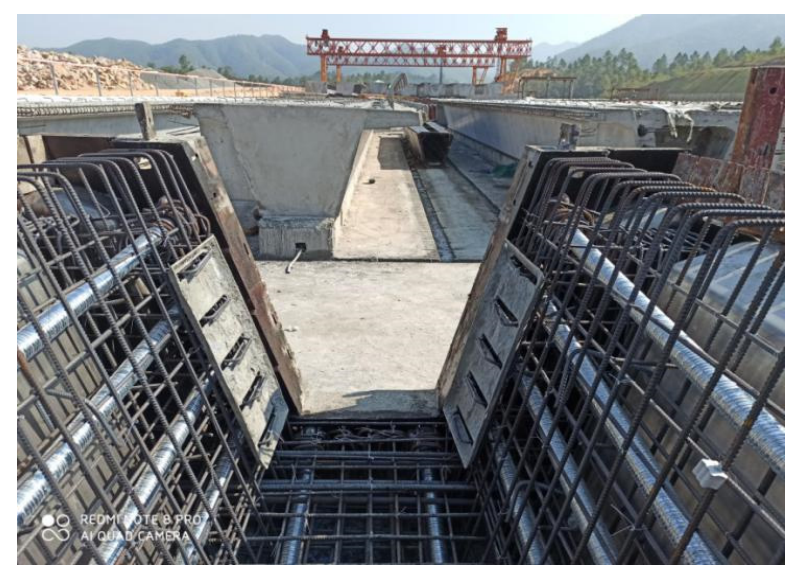

Figure 3. Anchor Plate Installation

\subsection{Steel Strand Cutting}

The steel strand must be laid underground in a flat, waterless and clean yard, and the laying length of the steel strand must be strictly in accordance with the drawings. Measure the length of the blanking from the end of the strand with a $50 \mathrm{~m}$ steel tape measure and mark the strand. Install the grinding wheel cutter, install limit mark at both ends of the discharging material and cut it. It is forbidden to cut the steel strand by arc welding. After cutting the steel strand with a grinding wheel cutter, the second material is laid according to the blanking size correctly. 


\subsection{Steel Strand Bundling}

The steel strands are taken out and laid out straight in accordance with the quantity required on the drawings. The strands must be laid parallel to each other and must not be crossed. They must be wired from the middle to both ends every $1 \mathrm{~m}$ and numbered. After bundling, they should be uniformly numbered and listed, and stacked neatly by category for use.

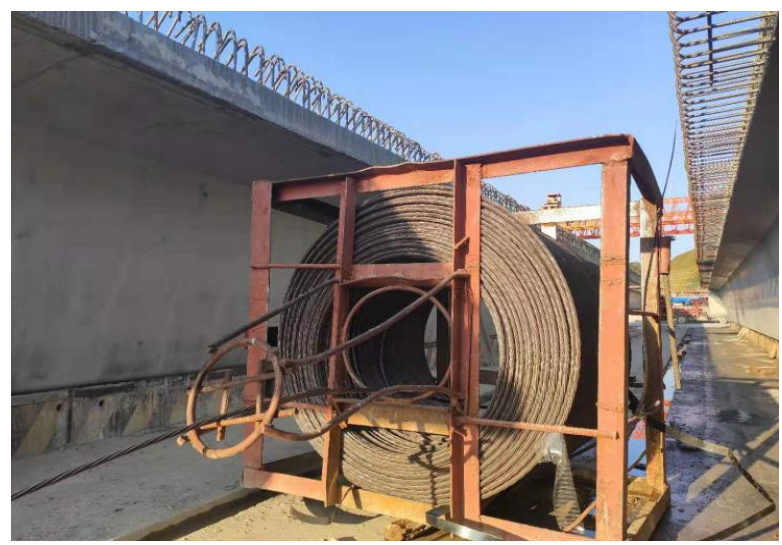

Figure 4. The winch pulls out the steel bundle

\subsection{Steel Strand Threading}

Check whether the pipeline is unblocked before threading. If blockage occurs, measures must be taken to unblock the pipeline. The ends of the steel strands must be tapered and wrapped so that they can be pulled by hand or by a hoist and threaded after concrete placement. During bundle threading, a lead is threaded into the pipeline and the steel wire is pulled out by the lead. The other end of the wire is connected with the steel bundle tow and the steel bundle is pulled out by a winch.

\subsection{Preparation Before Stretching}

1) Inspect the steel strands before tensioning to ensure that there is no rust and hard damage.

2) Test the strength of the concrete block before tension. Tension can only be carried out if the strength of the concrete block reaches $85 \%$ or more and it has more than 7 days of age.

3) Calibrate the jack, oil pump and pressure gauge before tensioning. The jack corresponding to the oil gauge needs to be checked every 300 times or within two months.

4) Group the tensioning equipment, the equipment with different group numbers shall not be mixed.

\subsection{Stretching}

Taking the $30 \mathrm{~m}$ small box girder as an example, the tension procedure is:

$$
0 \longrightarrow 10 \% \sigma \text { con } \rightarrow 20 \% \sigma \text { con } \rightarrow 100 \% \sigma \text { con. }
$$

Anchoring under load for $5 \mathrm{~min}$.

Prestress tension is constructed by double-control method, which means tension is carried out by controlled stress and checked by elongation of steel strand. When measuring the elongation of steel strand, it should be noted that the elongation of $0 \rightarrow 10 \% \sigma$ con should not be measured directly, but should be calculated, i.e. the elongation of $10 \% \sigma \mathrm{con} \rightarrow 20 \% \sigma$ con should be taken as the elongation of $0 \rightarrow 10 \% \sigma c o n$. The actual elongation of the steel strands at both ends is compared with the theoretical elongation with an error of $<6 \%$, otherwise the tension should be carried out after finding out the cause (the following steps can be taken: re-calibrate the pressure gauge and corresponding jack; perform elastic modulus test on the steel strands; re-tension the steel strands after tensioning; and coat the steel strands with lubricant to reduce friction loss).

Measure the Camber of the beam immediately after stretching, measure the camber of the beam in $1 \mathrm{~d}, 3 \mathrm{~d}, 7 \mathrm{~d}$, $30 \mathrm{~d}$ and $60 \mathrm{~d}$, accord with the design value.

\section{Points for attention}

Three pairs of holes, anchor plates and Jacks should be made in the course of stretching so that the stretching can proceed smoothly without increasing the friction loss of the holes

The two ends are synchronously and symmetrically tensioned, and the elongation of the two ends is basically the same in tensioning engineering. If one end of the oil pump is too fast and the other end of the oil pump is too slow, let the one end of the oil pump be stopped first and then start, try to keep consistent;

Pay Special attention to safety in tension work, because prestress has a lot of energy, in case the steel strand is broken or the energy is released rapidly after the anchor and Jack Fail, it may cause great harm. Therefore, personnel should not stand at both ends of the Steel Strand during the tensioning operation, and should be installed behind the operating platform protective devices.

\section{Application example}

The precast Beam Yard in our department has realized intelligent tension, which is not influenced by man-made and environmental factors, and the tension process factors such as control stopping point, loading rate, holding time, etc.

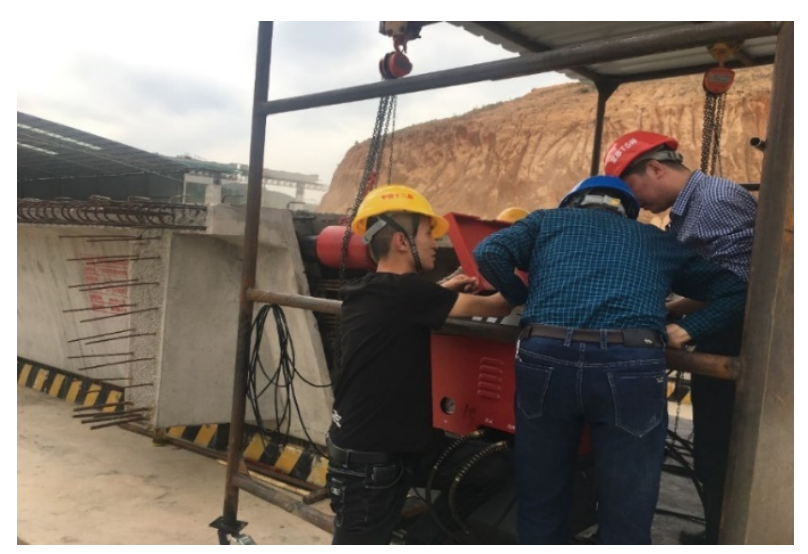

Figure 5. Construction process 
Evaluation of intelligent tensioning system:

(1) The tension control precision is high, the load is uniform and stable, and the tension deviation can be controlled within $\pm 1.5 \%$.

(2) The results show that the tension data is true and reliable, and the relevant charts are automatically recorded; each tension stroke is executed accurately without interference of human factors.

(3) It can reach $\pm 2 \%$ of the standard.

(4) With strong traceability, it is easy to analyze and study the problems in the tensioning process. Further improve the quality of components and construction management.

\section{Conclusion}

The use of intelligent tensioning equipment in domestic engineering construction began in 2010. Up to now, the application of intelligent tensioning system has entered a period of rapid growth. The number of equipment manufacturers has increased from a few in the initial stage to 30 or 40 . The scope of use includes highway bridges, municipal engineering bridges and railway bridges and other engineering projects in more than 20 provincial administrative regions. Whether from the quality of prestressed components or the quality of construction site management, the intelligent tensioning system has obvious advantages, which can better meet the requirements of construction specifications.

Intelligent tensioning refers to the completion of tensioning construction by computer intelligent control instead of manual control, which is widely used in bridge construction nowadays. The excellent quality of prestress will directly affect the stability and durability of the structure. However, the traditional tension construction, relying on the experience of manual operation, error rate is very high, can not guarantee the quality of prestressed construction.

\section{Acknowledgments}

Many thanks for the support of China Railway 12th Bureau Group Co., Ltd. and project management department of T5 bid of Zihui expressway for the application of this technology.

\section{References}

1. Chen, H.L. Analysis of Optimization Measures for Longitudinal Prestressed Tensioning Process of Long Span PC Beam Bridge. Highway Engineering, 2019(2): 131-137.

2. Li, Y.H, Fu, D.S Deng, X.D. (2020) Application of Stress Testing Technology Under Anchor in Continuous Rigid Frame Bridge Construction. Highway, 2020(2): 120-124.

3. Luo, J.L, Li, G.H. (2019) Study on The Influence of Tensioning Technology on Creep of Prestressed
Concrete Bridge. Construction Technology, 2019(11): 28-31.

4. Lu, X.M, Qian, J. (2019) Identification Method of Steel Strand Tension Based on Acoustoelastic Effect. Science Technology and Engineering, 2019(13): 281286.

5. He, Y.M. (2016) Experimental Study on The Influence of Controlled Oil Temperature of Intelligent Bridge Tensiometer on Performance of Pressure Sensor. Journal of China \& Foreign Highway, 2016, 36(5): 166-168. 